\title{
Long range order for lattice dipoles
}

\author{
Alessandro Giuliani \\ Dipartimento di Matematica, Università di Roma Tre, \\ L.go S. Leonardo Murialdo 1, 00146 Roma Italy
}

(Dated: 25 March 2008)

\begin{abstract}
We consider a system of classical Heisenberg spins on a cubic lattice in dimensions three or more, interacting via the dipole-dipole interaction. We prove that at low enough temperature the system displays orientational long range order, as expected by spin wave theory. The proof is based on reflection positivity methods. In particular, we demonstrate a previously unproven conjecture on the dispersion relation of the spin waves, first proposed by Fröhlich and Spencer, which allows one to apply infrared bounds for estimating the long distance behavior of the spin-spin correlation functions.
\end{abstract}

\section{INTRODUCTION}

Recent advances in film growth techniques, in the control of spin-spin interactions and in the ability to characterize magnetic materials have revived interest in the low temperature physics of magnetic systems. Both experimental and theoretical studies have revealed several unusual properties of magnetic films, such as spontaneous formation of striped patterns, reorientation transitions (in temperature and in the sample thickness), increase of the static magnetization with increasing temperature, just to mention a few [7]. It is believed that an essential role in determining the nature and morphology of the ordered state is played by the dipolar interaction. Unfortunately, its long-range nature and its anisotropic character make many standard theoretical methods and numerical algorithms inapplicable. It is therefore not surprising that, for instance, existence of long range order in 2D continuous spin systems interacting via a pure dipole-dipole interaction at low temperatures is still a matter of discussion, even at a heuristic level. In fact, the case of $2 \mathrm{D}$ lattice dipoles is a paradigmatic example of a system where the Mermin-Wagner theorem cannot be applied, spin-wave theory does not provide any resolutive answer and numerical simulations are difficult because of the slow relaxation dynamics associated with the long-range nature of the interaction. New and more sophisticated methods, such as renormalized spin wave theory [5,6] or block spin reflection positivity [13], are required to deal with this class of systems.

At a rigorous level, even widely accepted results, such as the existence of long range orientational order in three dimensional lattice dipole systems or the existence of the infinite volume Gibbs state for any given domain shape are yet to be fully proved. Many fundamental contributions to the rigorous theory of lattice dipole systems (and more generally of dipole gases on the lattice or in the continuum) date back to the 80 's. The use of several different techniques, such as reflection positivity, correlation inequalities, cluster expansion, renormalization group, allowed people to rigorously prove, e.g., no-screening theorems at any activity and temperature $[10,15]$, analyticity of the pressure at small activities $[1,2,4,12]$, existence of a scaling limit (Gaussian free field) for lattice dipoles at small activities [14] and the existence of the thermodynamic limit in the continuum in three or more dimensions [9], see [3] for a comprehensive review of these results. In a seminal paper, Fröhlich and Spencer [10], among several other results, proved existence of long range order for a system of discrete lattice dipoles in two or more dimensions. They also described a proof for the physically relevant case of continuous dipoles on the cubic lattice in three or more dimensions. However, in the case of infinite-range interaction, they could not give a complete proof, and their argument was based on an unproven conjecture on the dispersion relation of the spin waves. In this paper we will give a complete proof of long range order for classical dipoles on a cubic lattice in three or more dimensions, proving in particular the aforementioned Fröhlich-Spencer conjecture. The proof is based on reflection positivity and it extends ideas proposed in [10]. 


\section{MAIN RESULTS}

Let $\Lambda$ be a periodic box in $\mathbb{Z}^{d}$, viewed as the restriction of a periodic box in $\mathbb{R}^{d}$ to $\mathbb{Z}^{d}$. We assume that $\Lambda$ is of side $2 L$, with $L$ even, and we write: $\Lambda=[-L+1, \ldots, L]^{d}$. We consider the following Hamiltonian:

$$
H_{\Lambda}=\sum_{i, j=1}^{d} \sum_{\mathbf{x}, \mathbf{y} \in \Lambda} S_{\mathbf{x}}^{i} W_{i j}(\mathbf{x}-\mathbf{y}) S_{\mathbf{y}}^{j}
$$

where $\vec{S}_{\mathbf{x}}$ is a unit vector and $\left\{S_{\mathbf{x}}^{i}\right\}_{i=1, \ldots, d}$ are its components. Moreover, denoting the Yukawa potential by

$$
Y_{\varepsilon}(\mathbf{x})=\int \frac{d \mathbf{k}}{(2 \pi)^{d}} \frac{e^{i \mathbf{k x}}}{\mathbf{k}^{2}+\varepsilon^{2}}
$$

the interaction matrix $W(\mathbf{x})$ is defined as:

$$
W_{i j}(\mathbf{x})=\sum_{\mathbf{n} \in \mathbb{Z}^{d}}\left(-\partial_{i} \partial_{j}\right) Y_{\varepsilon_{\Lambda}}(\mathbf{x}+2 \mathbf{n} L), \quad \mathbf{x} \neq \mathbf{0}
$$

and $W_{i j}(\mathbf{0})=\sum_{\mathbf{n} \neq \mathbf{0}}\left(-\partial_{i} \partial_{j}\right) Y_{\varepsilon_{\Lambda}}(2 \mathbf{n} L)$. The parameter $\varepsilon_{\Lambda}$ is an infrared regulator that is sent to zero in the thermodynamic limit, i.e., $\lim _{|\Lambda| \rightarrow \infty} \varepsilon_{\Lambda}=0$.

For any fixed $\Lambda$ and $\beta>0$, let us denote by $\langle\cdot\rangle_{\beta, \Lambda}$ the Gibbs expectation given by the probability measure $Z_{\beta, \Lambda}^{-1} \prod_{\mathbf{x} \in \Lambda} d \mu\left(\vec{S}_{\mathbf{x}}\right) e^{-\beta H_{\Lambda}}$, with $d \mu(\vec{S})$ the uniform measure on the unit sphere and $Z_{\beta, \Lambda}$ the obvious normalization factor. For any $\mathbf{x} \in \Lambda$, given the unit vector $\vec{S}_{\mathbf{x}}$, we define:

$$
\sigma_{\mathbf{x}}^{i}=(-1)^{\mathbf{x}+x_{i}} S_{\mathbf{x}}^{i}
$$

and denote by $\vec{\sigma}_{\mathbf{x}}$ the unit vector with components $\sigma_{\mathbf{x}}^{i}$. Our main result is the following.

Theorem 1 (Existence of orientational Long Range Order). If $d \geq 3$, there exists $\beta_{d}>0$ such that, if $\beta>\beta_{d}$, in the thermodynamic limit,

$$
\lim _{|\Lambda| \rightarrow \infty} \frac{1}{|\Lambda|^{2}} \sum_{\mathbf{x}, \mathbf{y} \in \Lambda}\left\langle\vec{\sigma}_{\mathbf{x}} \cdot \vec{\sigma}_{\mathbf{y}}\right\rangle_{\beta, \Lambda} \geq c_{d}(\beta)>0
$$

with $c_{d}(\beta)$ a suitable positive function, vanishing at $\beta=\beta_{d}$.

Using the methods of [9], it can be proved that the state $\langle\cdot\rangle_{\beta, \Lambda}$ admits a thermodynamic limit, which we will denote by $\langle\cdot\rangle_{\beta}$. The theorem above implies that, for $\beta>\beta_{d}$, the infinite volume Gibbs state $\langle\cdot\rangle_{\beta}$ is not an extremal Gibbs state. From the assumed symmetry of $\langle\cdot\rangle_{\beta, \Lambda}$ under exchanges of the coordinate axes, and by the general theory of decomposition into extremal states, it follows that $\langle\cdot\rangle_{\beta}$ is a mixture of at least $2 d$ extremal Gibbs states (pure phases $),\langle\cdot\rangle_{\beta}^{(\lambda)}$, which break rotational invariance and are characterized by

$$
\left\langle S_{\mathbf{x}}^{i}\right\rangle_{\beta}^{(\lambda)}=(-1)^{\mathbf{x}+x_{i}} v_{\lambda}^{i}
$$

where $\left\{\vec{v}_{\lambda}: \lambda=1, \ldots, 2 d, \ldots\right\}$ are vectors obtained from some vector $\vec{v}_{0} \in \mathbb{R}^{d}$ by applying arbitrary rotations around the origin which leave the unit cube centered at the origin invariant.

The rest of the paper will be devoted to the proof of Theorem 1. As mentioned in the introduction, we will follow the same strategy proposed by Fröhlich and Spencer in [10] and we will extend their reflection positivity method to prove, in particular, their Conjecture 7.9 [10]. In order to present a self-contained proof, we shall reproduce below some of the statements already proved in [10], including a construction of the ground states of (2.1). 


\section{PROOF OF THEOREM 1}

\section{A. Reflection Positivity.}

Let us recall the notion of reflection positivity, adapted to the present case. If $\vec{S}$ is a unit vector, define

$$
\left(R_{i} \vec{S}\right)^{j}=(-1)^{1-\delta_{i, j}} S^{j} .
$$

Let $\pi_{i}$ be a pair of planes perpendicular to the $i$-th direction, midway in between two lattice planes and bisecting $\Lambda$ into two pieces $\Lambda_{+}$and $\Lambda_{-}$of equal size. Let $r_{i}$ denote reflection of sites with respect to $\pi_{i}$. Clearly $r_{i} \Lambda_{-}=\Lambda_{+}$. We define

$$
\left(\theta_{i} \vec{S}\right)_{\mathbf{x}}=R_{i} \vec{S}_{r_{i} \mathbf{x}}
$$

Let $\mathcal{S}_{ \pm}=\left\{\vec{S}_{\mathbf{x}}\right\}_{\mathbf{x} \in \Lambda_{ \pm}}$. If $A$ is a function of $\mathcal{S}_{+}$we set

$$
\left(\theta_{i} A\right)\left(\mathcal{S}_{-}\right)=A\left(\left\{\left(\theta_{i} \vec{S}\right)_{\mathbf{x}}\right\}_{\mathbf{x} \in \Lambda_{-}}\right)
$$

We shall say that the expectation $\langle\cdot\rangle_{\beta, \Lambda}$ is reflection positive (RP) iff, for an arbitrary function $A$ of $\mathcal{S}_{+}$

$$
\left.\overline{\left\langle\theta_{i} A\left(\mathcal{S}_{-}\right)\right.} A\left(\mathcal{S}_{+}\right)\right\rangle_{\beta, \Lambda} \geq 0
$$

for $i=1, \ldots, d$. As discussed in [8] and in [10], a sufficient condition for (3.10) to hold is the following. Let $\vec{\rho}: \mathbb{R}^{d} \rightarrow \mathbb{R}^{d}$ be an arbitrary $\mathbb{R}^{d}$ valued function of $\mathbb{R}^{d}$. Assume that

$$
-\sum_{l, m=1}^{d} \int_{\substack{x_{i}>0 \\ y_{i}<0}} \rho^{l}(\mathbf{x})\left(\theta_{i} \rho\right)^{m}(\mathbf{y})\left(-\partial_{l} \partial_{m}\right) Y_{\varepsilon_{\Lambda}}(\mathbf{x}-\mathbf{y}) \geq 0
$$

for all $\vec{\rho}(\mathbf{x})$ and $i=1, \ldots, d$. Then $\langle\cdot\rangle_{\beta, \Lambda}$ is RP.

In our context the proof of (3.11) proceeds as follows. For definiteness, let us assume that $i=1$. Let $x_{1}>0$ and let us rewrite

$$
Y_{\varepsilon_{\Lambda}}(\mathbf{x})=\int \frac{d \mathbf{k}}{(2 \pi)^{d}} \frac{e^{i \mathbf{k x}}}{\mathbf{k}^{2}+\varepsilon_{\Lambda}^{2}}=\frac{1}{2(2 \pi)^{d-1}} \int \frac{d \mathbf{k}_{\perp}}{\sqrt{\mathbf{k}_{\perp}^{2}+\varepsilon_{\Lambda}^{2}}} e^{i \mathbf{k}_{\perp} \cdot \mathbf{x}_{\perp}} e^{-\left|x_{1}\right| \sqrt{\mathbf{k}_{\perp}^{2}+\varepsilon_{\Lambda}^{2}}}
$$

where in the last expression $\mathbf{k}_{\perp}=\left(k_{2}, \ldots, k_{d}\right)$ and similarly for $\mathbf{x}_{\perp}$. If $x_{1}>y_{1}$, given any two functions $\rho_{1}(\mathbf{x}), \rho_{2}(\mathbf{x})$ :

$$
\begin{aligned}
& \sum_{l, m=1}^{d} \rho_{1}^{l}(\mathbf{x}) \rho_{2}^{m}(\mathbf{y}) \partial_{l} \partial_{m} Y_{\varepsilon_{\Lambda}}(\mathbf{x}-\mathbf{y})=\frac{1}{2(2 \pi)^{d-1}} \int \frac{d \mathbf{k}_{\perp}}{\sqrt{\mathbf{k}_{\perp}^{2}+\varepsilon_{\Lambda}^{2}}} e^{i \mathbf{k}_{\perp} \cdot\left(\mathbf{x}_{\perp}-\mathbf{y}_{\perp}\right)} e^{-\left(x_{1}-y_{1}\right) \sqrt{\mathbf{k}_{\perp}^{2}+\varepsilon_{\Lambda}^{2}}} \\
& \cdot\left(\rho_{1}^{1}(\mathbf{x}) \sqrt{\mathbf{k}_{\perp}^{2}+\varepsilon_{\Lambda}^{2}}-i \mathbf{k}_{\perp} \varrho_{1}^{\perp}(\mathbf{x})\right)\left(\rho_{2}^{1}(\mathbf{y}) \sqrt{\mathbf{k}_{\perp}^{2}+\varepsilon_{\Lambda}^{2}}-i \mathbf{k}_{\perp} \varrho_{2}^{\perp}(\mathbf{y})\right)
\end{aligned}
$$

where $\varrho_{1}^{\perp}=\left(\rho_{1}^{2}, \ldots, \rho_{1}^{d}\right)$ and $\varrho_{2}^{\perp}=\left(\rho_{2}^{2}, \ldots, \rho_{2}^{d}\right)$.

Using this expression we find that, if $i=1,(3.11)$ can be rewritten as

$$
\begin{aligned}
& \frac{1}{2(2 \pi)^{d-1}} \int_{x_{1}>0} d \mathbf{x} \int_{y_{1}>0} d \mathbf{y} \int \frac{d \mathbf{k}_{\perp}}{\sqrt{\mathbf{k}_{\perp}^{2}+\varepsilon_{\Lambda}^{2}}} e^{i \mathbf{k}_{\perp} \cdot\left(\mathbf{x}_{\perp}-\mathbf{y}_{\perp}\right)} e^{-\left(x_{1}+y_{1}\right) \sqrt{\mathbf{k}_{\perp}^{2}+\varepsilon_{\Lambda}^{2}}} . \\
& \cdot\left(\rho^{1}(\mathbf{x}) \sqrt{\mathbf{k}_{\perp}^{2}+\varepsilon_{\Lambda}^{2}}-i \mathbf{k}_{\perp} \varrho^{\perp}(\mathbf{x})\right)\left(\rho^{1}(\mathbf{y}) \sqrt{\mathbf{k}_{\perp}^{2}+\varepsilon_{\Lambda}^{2}}+i \mathbf{k}_{\perp} \varrho^{\perp}(\mathbf{y})\right)
\end{aligned}
$$

that is clearly non-negative. Let us remark that condition (3.11) is equivalent to the statement that $H_{\Lambda}=H_{\Lambda}\left(\mathcal{S}_{-}, \mathcal{S}_{+}\right)$can be rewritten in the following form [8]:

$$
H_{\Lambda}\left(\mathcal{S}_{-}, \mathcal{S}_{+}\right)=H_{+}\left(\mathcal{S}_{+}\right)+\theta_{i} H_{+}\left(\mathcal{S}_{-}\right)-\int d \rho(\mathbf{q}) \overline{\theta_{i} C_{\mathbf{q}}\left(\mathcal{S}_{-}\right)} C_{\mathbf{q}}\left(\mathcal{S}_{+}\right),
$$


for a suitable positive measure $d \rho$. In our case,

$$
H_{+}\left(\mathcal{S}_{+}\right)=\sum_{i, j=1}^{d} \sum_{\mathbf{x}, \mathbf{y} \in \Lambda_{+}} S_{\mathbf{x}}^{i} W_{i j}(\mathbf{x}-\mathbf{y}) S_{\mathbf{y}}^{j} .
$$

Moreover, if $i=1$ and $\Lambda_{+}=[1, \ldots, L] \times[-L+1, \ldots, L]^{d-1}, \mathbf{q}$ is a $(d-1)$-dimensional vector and, defining $\mathbf{S}_{\mathbf{x}}^{\perp}=\left(S_{\mathbf{x}}^{2}, \ldots, S_{\mathbf{x}}^{d}\right)$,

$$
\begin{aligned}
& C_{\mathbf{q}}\left(\mathcal{S}_{+}\right)=\sum_{\mathbf{x} \in \Lambda_{-}}\left[S_{\mathbf{x}}^{1} \sqrt{\mathbf{q}^{2}+\varepsilon_{\Lambda}^{2}}-i \mathbf{q} \cdot \mathbf{S}_{\mathbf{x}}^{\perp}\right] e^{i \mathbf{q} \cdot \mathbf{x}_{\perp}} e^{-x_{1} \sqrt{\mathbf{q}^{2}+\varepsilon_{\Lambda}^{2}}} \\
& d \rho(\mathbf{q})=\frac{d \mathbf{q}}{2(2 \pi)^{d-1}} \frac{e^{-\sqrt{\mathbf{q}^{2}+\varepsilon_{\Lambda}^{2}}}}{\sqrt{\mathbf{q}^{2}+\varepsilon_{\Lambda}^{2}}} .
\end{aligned}
$$

If $i>1$ and/or $\Lambda_{+}$is different from $[1, \ldots, L] \times[-L+1, \ldots, L]^{d-1}$, analogous expressions for $C_{\mathbf{q}}$ and $d \rho(\mathbf{q})$ will be valid.

\section{B. Ground states.}

Let us now show how reflection positivity allows us to construct the ground states of (2.1). The key remark is that the positive measure $d \rho(\mathbf{q})$ in (3.15) induces the definition of a scalar product between spin configurations in $\Lambda_{+}$. In particular, combining the Cauchy-Schwarz inequality and the inequality of arithmetic and geometric means, we find that

$$
\begin{aligned}
\int d \rho(\mathbf{q}) & \overline{\theta_{i} C_{\mathbf{q}}\left(\mathcal{S}_{-}\right)} C_{\mathbf{q}}\left(\mathcal{S}_{+}\right) \leq \\
& \leq\left[\int d \rho(\mathbf{q}) \overline{\theta_{i} C_{\mathbf{q}}\left(\theta_{i} \mathcal{S}_{+}\right)} C_{\mathbf{q}}\left(\mathcal{S}_{+}\right)\right]^{1 / 2} \cdot\left[\int d \rho(\mathbf{q}) \overline{\theta_{i} C_{\mathbf{q}}\left(\mathcal{S}_{-}\right)} C_{\mathbf{q}}\left(\theta_{i} \mathcal{S}_{-}\right)\right]^{1 / 2} \\
& \leq \int d \rho(\mathbf{q}) \overline{\theta_{i} C_{\mathbf{q}}\left(\theta_{i} \mathcal{S}_{+}\right)} C_{\mathbf{q}}\left(\mathcal{S}_{+}\right)+\int d \rho(\mathbf{q}) \overline{\theta_{i} C_{\mathbf{q}}\left(\mathcal{S}_{-}\right)} C_{\mathbf{q}}\left(\theta_{i} \mathcal{S}_{-}\right)
\end{aligned}
$$

If we insert this estimate in (3.15), we find that

$$
H_{\Lambda}\left(\mathcal{S}_{-}, \mathcal{S}_{+}\right) \geq \frac{1}{2} H_{\Lambda}\left(\theta_{i} \mathcal{S}_{+}, \mathcal{S}_{+}\right)+\frac{1}{2} H_{\Lambda}\left(\mathcal{S}_{-}, \theta_{i} \mathcal{S}_{-}\right)
$$

that is, either $\left\{\theta_{i} \mathcal{S}_{+}, \mathcal{S}_{+}\right\}$or $\left\{\mathcal{S}_{-}, \theta_{i} \mathcal{S}_{-}\right\}$has lower energy than $\left\{\mathcal{S}_{-}, \mathcal{S}_{+}\right\}$. If we keep reflecting in different planes, using the chessboard estimate (see Theorem 4.1 in [8]), we find that the energy $H_{\Lambda}(\mathcal{S})$ of a generic configuration of spins $\mathcal{S}=\left\{\vec{S}_{\mathbf{x}}\right\}_{\mathbf{x} \in \Lambda}$ is bounded below by $|\Lambda|^{-1} \sum_{\mathbf{x}_{0} \in \Lambda} H_{\Lambda}\left(\mathcal{S}_{\mathbf{x}_{0}}\right)$, where the spin at $\mathbf{x}$ in the configuration $\mathcal{S}_{\mathbf{x}_{0}}$ is given by $(-1)^{\mathbf{x}}(-1)^{x_{i}} S_{\mathbf{x}_{0}}^{i}, i=1, \ldots, d$. We now show that $H_{\Lambda}\left(\mathcal{S}_{\mathbf{x}_{0}}\right)$ is independent of $\vec{S}_{\mathbf{x}_{0}}$. Note that this is not apriori obvious, since the Hamiltonian is not invariant under global rotations. Let $\vec{S}_{\mathbf{x}_{0}}=(\sin \theta \cos \phi, \sin \theta \sin \varphi, \cos \theta)$. Then

$$
\begin{aligned}
H_{\Lambda}\left(\mathcal{S}_{\mathbf{x}_{0}}\right)=-\sum_{\substack{\mathbf{x} \in \Lambda \\
\mathbf{y} \in \mathbb{Z}^{d} \backslash \mathbf{x}}}(-1)^{\mathbf{x}-\mathbf{y}} & \left\{\sum_{i=1}^{d}(-1)^{x_{i}-y_{i}}\left(S_{\mathbf{x}_{0}}^{i}\right)^{2} \partial_{i}^{2} Y_{\varepsilon_{\Lambda}}(\mathbf{x}-\mathbf{y})+\right. \\
& \left.+\sum_{i \neq j=1}^{d}(-1)^{x_{i}}(-1)^{y_{j}} S_{\mathbf{x}_{0}}^{i} S_{\mathbf{x}_{0}}^{j} \partial_{i} \partial_{j} Y_{\varepsilon_{\Lambda}}(\mathbf{x}-\mathbf{y})\right\}
\end{aligned}
$$

In (3.14) the summations over $\mathbf{x}$ and $\mathbf{x}-\mathbf{y}$ factor, both for the term in the first line and for the one in the second line. In particular the term in the second line is zero after summation. So we are left with

$$
H_{\Lambda}\left(\mathcal{S}_{\mathbf{x}_{0}}\right)=e_{0}|\Lambda|, \quad e_{0}=\sum_{\mathbf{x} \neq \mathbf{0}}(-1)^{\mathbf{x}+x_{1}}\left(-\partial_{1}^{2}\right) Y_{\varepsilon_{\Lambda}}(\mathbf{x})
$$

where we used that $\sum_{i}\left(S_{\mathbf{x}_{0}}^{i}\right)^{2}=1$ and the rotation symmetry of $Y_{\varepsilon_{\Lambda}}(\mathbf{x})$. 


\section{Infrared bounds.}

In this section we will extend the ideas used above to construct the ground states, and we will derive lower bounds on the Fourier transform of $\left\langle\vec{\sigma}_{\mathbf{x}} \cdot \vec{\sigma}_{\mathbf{y}}\right\rangle_{\beta, \Lambda}$ (also known as infrared bounds [11]). This will conclude the proof of Theorem 1. The main ingredients that we will need are Gaussian domination [11] and a refinement of the estimates on the dispersion relation of the spin waves, including a proof of Conjecture 7.9 in [10].

As a first step we map the spin system in (2.1) onto a ferromagnetic spin system via the mapping $\vec{S}_{\mathbf{x}} \longleftrightarrow \vec{\sigma}_{\mathbf{x}}$ defined by (2.4). In terms of the $\vec{\sigma}$ 's, the Hamiltonian can be rewritten as

$$
H_{\Lambda}^{\prime}=\sum_{i, j=1}^{d} \sum_{\mathbf{x}, \mathbf{y} \in \Lambda} \sigma_{\mathbf{x}}^{i} W_{i j}^{\prime}(\mathbf{x}, \mathbf{y}) \sigma_{\mathbf{y}}^{j}
$$

with $W_{i j}^{\prime}(\mathbf{x}, \mathbf{y})=(-1)^{\mathbf{x}-\mathbf{y}}(-1)^{x_{i}+y_{j}} W_{i j}(\mathbf{x}-\mathbf{y})$. Note that $W^{\prime}(\mathbf{x}, \mathbf{y})$ is not translation invariant. The discussion above shows that the ground states of $H_{\Lambda}^{\prime}$ are spin configurations with spins all pointing in the same direction in space. Moreover $H_{\Lambda}^{\prime}$ is reflection positive with respect to the ferromagnetic reflection $\left(\theta_{i}^{\prime} \vec{\sigma}\right)_{\mathbf{x}}=\vec{\sigma}_{r_{i} \mathbf{x}}$. We rewrite $H_{\Lambda}^{\prime}$ in the form

$$
H_{\Lambda}^{\prime}=-\frac{1}{2} \sum_{i, j=1}^{d} \sum_{\mathbf{x}, \mathbf{y} \in \Lambda}\left(\sigma_{\mathbf{x}}^{i}-\sigma_{\mathbf{y}}^{i}\right) W_{i j}^{\prime}(\mathbf{x}, \mathbf{y})\left(\sigma_{\mathbf{x}}^{j}-\sigma_{\mathbf{y}}^{j}\right)+\sum_{i, j=1}^{d} \sum_{\mathbf{x} \in \Lambda} \sigma_{\mathbf{x}}^{i} \sigma_{\mathbf{x}}^{j} \sum_{\mathbf{y}} W_{i j}^{\prime}(\mathbf{x}, \mathbf{y})
$$

Note that $\sum_{\mathbf{y}} W_{i j}^{\prime}(\mathbf{x}, \mathbf{y})=e_{0} \mathbb{1}$, so that

$$
H_{\Lambda}^{\prime}=-\frac{1}{2} \sum_{i, j=1}^{3} \sum_{\mathbf{x}, \mathbf{y} \in \Lambda}\left(\sigma_{\mathbf{x}}^{i}-\sigma_{\mathbf{y}}^{i}\right) W_{i j}^{\prime}(\mathbf{x}, \mathbf{y})\left(\sigma_{\mathbf{x}}^{j}-\sigma_{\mathbf{y}}^{j}\right)+e_{0}|\Lambda|
$$

Let us now define:

$$
K_{\beta, \Lambda}(\underline{\vec{h}})=\left\langle\exp \left\{\frac{\beta}{2} \sum_{i, j=1}^{d} \sum_{\mathbf{x}, \mathbf{y} \in \Lambda}\left(\sigma_{\mathbf{x}}^{i}-\sigma_{\mathbf{y}}^{i}-h_{\mathbf{x}}^{i}+h_{\mathbf{y}}^{i}\right) W_{i j}^{\prime}(\mathbf{x}, \mathbf{y})\left(\sigma_{\mathbf{x}}^{j}-\sigma_{\mathbf{y}}^{j}-h_{\mathbf{x}}^{j}+h_{\mathbf{y}}^{j}\right)\right\}\right\rangle_{0, \Lambda}
$$

for $\vec{h}_{\mathbf{x}}, \mathbf{x} \in \Lambda$, real vectors. The chessboard estimate (see Theorem 4.1 in [8]) shows that $K_{\beta, \Lambda}(\underline{\vec{h}}) \leq K_{\beta, \Lambda}(\underline{\overrightarrow{0}})=K_{\beta, \Lambda}$ (Gaussian domination). This implies $\left.\frac{d^{2}}{d \lambda^{2}} K_{\beta, \Lambda}(\lambda \underline{\vec{h}})\right|_{\lambda=0} \leq 0$, that is $\beta\left\langle\left|\sum_{i, j=1}^{d} \sum_{\mathbf{x}, \mathbf{y} \in \Lambda}\left(h_{\mathbf{x}}^{i}-h_{\mathbf{y}}^{i}\right) W_{i j}^{\prime}(\mathbf{x}, \mathbf{y})\left(\sigma_{\mathbf{x}}^{j}-\sigma_{\mathbf{y}}^{j}\right)\right|^{2}\right\rangle_{\beta, \Lambda}^{\prime} \leq-\sum_{i, j=1}^{d} \sum_{\mathbf{x}, \mathbf{y} \in \Lambda}\left(h_{\mathbf{x}}^{i}-h_{\mathbf{y}}^{i}\right)^{*} W_{i j}^{\prime}(\mathbf{x}, \mathbf{y})\left(h_{\mathbf{x}}^{j}-h_{\mathbf{y}}^{j}\right)$

where $\langle\cdot\rangle_{\beta, \Lambda}^{\prime}$ is the average with statistical weight $Z_{\beta, \Lambda}^{-1} e^{-\beta H_{\Lambda}^{\prime}}$. Note that $(3.26)$ holds apriori for real $\vec{h}_{\mathbf{x}}$, but it extends to complex vectors [8]. In terms of the original spins, (3.26) reads

$2 \beta\left\langle\left|\sum_{i, j=1}^{d} \sum_{\mathbf{x}, \mathbf{y} \in \Lambda} h_{\mathbf{x}}^{i} W_{i j}(\mathbf{x}-\mathbf{y}) S_{\mathbf{y}}^{j}-e_{0} \sum_{\mathbf{x} \in \Lambda} \vec{h}_{\mathbf{x}} \cdot \vec{S}_{\mathbf{x}}\right|^{2}\right\rangle_{\beta, \Lambda} \leq \sum_{i, j=1}^{d} \sum_{\mathbf{x}, \mathbf{y} \in \Lambda}\left(h_{\mathbf{x}}^{i}\right)^{*} W_{i j}(\mathbf{x}-\mathbf{y}) h_{\mathbf{y}}^{j}-e_{0} \sum_{\mathbf{x} \in \Lambda}\left|\vec{h}_{\mathbf{x}}\right|^{2}$

for some new vectors $\vec{h}_{\mathbf{x}}$ (that we are denoting by the same symbol for simplicity). Let $\mathbf{p} \in$ $\mathcal{D}_{L} \equiv\left\{\frac{2 \pi}{2 L} \mathbf{m}, 0 \leq m_{1}, m_{2}, m_{3}<2 L\right\}$ and let $\hat{W}_{i j}(\mathbf{p})=\sum_{\mathbf{x} \in \Lambda} e^{i \mathbf{p x}} W_{i j}(\mathbf{x})$ denote the Fourier transform of $W(\mathbf{x})$. We shall also define

$$
\hat{S}_{\mathbf{p}}^{i}=\frac{1}{|\Lambda|^{1 / 2}} \sum_{\mathbf{x} \in \Lambda} e^{i \mathbf{p x}} S_{\mathbf{x}}^{i}, \quad Q_{i j}(\mathbf{p})=\left\langle\hat{S}_{\mathbf{p}}^{i} \hat{S}_{\mathbf{p}}^{j}\right\rangle_{\beta, \Lambda} .
$$

Given $\mathbf{p}$ such that $\hat{W}^{0}(\mathbf{p}) \equiv \hat{W}(\mathbf{p})-e_{0} \geq 0$ as a matrix and choosing $\vec{h}_{\mathbf{x}}=|\Lambda|^{-1 / 2} e^{-i \mathbf{p x}} \vec{v}$ in (3.27), we get:

$$
0 \leq \hat{W}^{0}(\mathbf{p}) Q(\mathbf{p}) \hat{W}^{0}(\mathbf{p}) \leq \frac{1}{2 \beta} \hat{W}^{0}(\mathbf{p})
$$


in the sense of an inequality between non-negative matrices. If, moreover, $\hat{W}^{0}(\mathbf{p})>0$, then

$$
0 \leq Q(\mathbf{p}) \leq \frac{1}{2 \beta} \hat{W}^{0}(\mathbf{p})^{-1}
$$

Eq.(3.29)-(3.30) are the key bounds. In order to make use of them, we need to study some properties of $\hat{W}^{0}(\mathbf{p})$, in particular we need to: (i) show that $\hat{W}^{0} \geq 0$; (ii) determine the set $S$ of momenta where $\hat{W}^{0}(\mathbf{p})$ has a vanishing eigenvalue; (iii) determine the behavior of its eigenvalues close to the zeros. Property (i) was prover in [10] (we shall reproduce the proof below). Moreover, in [10] it was conjectured (see Conjecture 7.9 in [10]) that, if $\boldsymbol{\pi}_{\ell}$ is the vector with components $\left(\boldsymbol{\pi}_{\ell}\right)^{j}=\pi\left(1-\delta_{\ell, j}\right)$, then $S=\left\{\boldsymbol{\pi}^{(\ell)}: \ell=1, \ldots, d\right\}$ and the eigenvalue $\lambda_{\ell}(\mathbf{p})$ vanishing at $\boldsymbol{\pi}_{\ell}$, satisfies $\lambda_{\ell}(\mathbf{p}) \geq c\left|\mathbf{p}-\boldsymbol{\pi}_{\ell}\right|^{2}$ close to $\boldsymbol{\pi}_{\ell}$, for some $c>0$. Our next goal will be to prove this conjecture.

Let us start with showing that $\hat{W}^{0}(\mathbf{p}) \geq 0$, for all p's. This is equivalent to the claim that, for any $\vec{v} \in \mathbb{R}^{d}$ and for $\vec{h}_{\mathbf{x}}=e^{i \mathbf{p x}} \vec{v}$,

$$
\sum_{i, j=1}^{d} \sum_{\mathbf{x}, \mathbf{y} \in \Lambda}\left(h_{\mathbf{x}}^{i}\right)^{*} W_{i j}^{0}(\mathbf{x}-\mathbf{y}) h_{\mathbf{y}}^{j} \geq 0
$$

By the chessboard estimate, the left hand side of (3.31) is bounded below by $|\Lambda|^{-1} \sum_{\mathbf{x}_{0} \in \Lambda}\left(H_{\Lambda}\left(\mathcal{H}_{\mathbf{x}_{0}}\right)-e_{0}|\vec{v}|^{2}\right)$, where the spin at $\mathbf{x}$ in the configuration $\mathcal{H}_{\mathbf{x}_{0}}$ is given by $(-1)^{\mathbf{x}}(-1)^{x_{i}} h_{\mathbf{x}_{0}}^{i}, i=1, \ldots, d$. Now note that $H_{\Lambda}\left(\mathcal{H}_{\mathbf{x}_{0}}\right)=e_{0}$, so the proof of $(3.31)$ is concluded. Note that if $\mathbf{p} \in S$ then $\hat{W}^{0}(\mathbf{p})=0$. We now want to get a more refined bound from below on $\hat{W}^{0}(\mathbf{p})$. In order to do this, we use the assumption that $L$ is even (so that the side of $\Lambda$ is divisible by 4 ) and we repeatedly reflect the left hand side of (3.31) in planes $\pi_{1}$ bisecting the horizontal bonds of the form $\left\{\left(2 m-1, \mathbf{x}_{\perp}\right),\left(2 m, \mathbf{x}_{\perp}\right)\right\}$. Finally we repeatedly reflect in all possible planes $\pi_{2}, \ldots, \pi_{d}$. The result is a bound from below of the form:

$$
\sum_{i, j=1}^{d} v^{i} \hat{W}_{i j}^{0}(\mathbf{p}) v^{j} \geq \frac{1}{|\Lambda|} \sum_{i, j=1}^{d} \sum_{\mathbf{x}, \mathbf{y} \in \Lambda}\left(u_{\mathbf{x}}^{i}\right)^{*} W_{i j}(\mathbf{x}-\mathbf{y}) u_{\mathbf{y}}^{j}-e_{0}|\vec{v}|^{2}
$$

with

$$
\begin{aligned}
& u_{\mathbf{x}}^{1}=e^{i \frac{p_{1}}{2} f_{1}\left(x_{1}\right)}(-1)^{\mathbf{x}+x_{1}} v_{1} \\
& u_{\mathbf{x}}^{i}=e^{i \frac{p_{1}}{2} f_{1}\left(x_{1}\right)}(-1)^{\mathbf{x}+x_{1}+x_{i}} f_{0}\left(x_{1}\right) v_{i}, \quad i>1
\end{aligned}
$$

where:

$$
f_{0}(x)=\left\{\begin{array}{ll}
1 & \text { if } x=4 k \\
1 & \text { if } x=4 k+1 \\
-1 & \text { if } x=4 k+2 \\
-1 & \text { if } x=4 k+3
\end{array}, \quad f_{1}(x)=f_{0}(x-1)\right.
$$

and $k \in \mathbb{Z}$. Given a lattice function $g(x)$ of a single variable with period 4 , we shall write $g(x)=\{g(0), g(1), g(2), g(3)\}$. Note that with this convention $f_{0}(x)=\{1,1,-1,-1\}$ and $f_{1}(x)=\{-1,1,1,-1\}$.

We now turn to the computation of the r.h.s. of (3.32). First of all, note that $\sum_{\mathbf{x}, \mathbf{y} \in \Lambda}\left(u_{\mathbf{x}}^{i}\right)^{*} W_{i j}(\mathbf{x}-\mathbf{y}) u_{\mathbf{y}}^{j}=0$ if $i \neq j$. This is because the double summation can be rewritten in the form $\sum_{\mathbf{x} \in \Lambda} \sum_{\mathbf{y} \in \mathbb{Z}^{d} \backslash \mathbf{x}} F_{i j}(\mathbf{x}, \mathbf{x}-\mathbf{y})$, for some function $F_{i j}(\mathbf{x}, \mathbf{x}-\mathbf{y})$ that is odd in $\left(x_{2}-y_{2}\right)$ and/or in $\left(x_{3}-y_{3}\right), \ldots,\left(x_{d}-y_{d}\right)$, depending on the specific matrix element. Let us consider $\sum_{\mathbf{x}, \mathbf{y} \in \Lambda}\left(u_{\mathbf{x}}^{1}\right)^{*} W_{11}(\mathbf{x}-\mathbf{y}) u_{\mathbf{y}}^{1}$. This double summation is equal to

$$
v_{1}^{2} \sum_{\mathbf{x} \in \Lambda} \sum_{\mathbf{y} \in \mathbb{Z}^{d} \backslash \mathbf{x}} e^{-i \frac{p_{1}}{2}\left(f_{1}\left(x_{1}\right)-f_{1}\left(y_{1}\right)\right)}(-1)^{\mathbf{x}-\mathbf{y}+\left(x_{1}-y_{1}\right)}\left(-\partial_{1}^{2}\right) Y_{\varepsilon_{\Lambda}}(\mathbf{x}-\mathbf{y})
$$

A computation shows that $f_{1}(x)-f_{1}(y)=f_{1}(x)\left[1-g_{0}(x-y)\right]+f_{0}(x) g_{1}(x-y)$, with $g_{0}(x)=$ $\{1,0,-1,0\}$ and $g_{1}(x)=g_{0}(x-1)$. Then we can rewrite $(3.35)$ as

$$
v_{1}^{2} \sum_{\mathbf{x} \in \Lambda} \sum_{\mathbf{y} \in \mathbb{Z}^{d} \backslash \mathbf{x}} e^{-i \frac{p_{1}}{2}\left\{f_{1}\left(x_{1}\right)\left[1-g_{0}\left(x_{1}-y_{1}\right)\right]+f_{0}\left(x_{1}\right) g_{1}\left(x_{1}-y_{1}\right)\right\}}(-1)^{\mathbf{x}-\mathbf{y}+\left(x_{1}-y_{1}\right)}\left(-\partial_{1}^{2}\right) Y_{\varepsilon_{\Lambda}}(\mathbf{x}-\mathbf{y})
$$


Performing the summation over $\mathbf{x}$ yields

$$
\begin{aligned}
& v_{1}^{2}|\Lambda| \sum_{\mathbf{x} \neq \mathbf{0}} \cos \left(\frac{p_{1}}{2}\left(1-g_{0}\left(x_{1}\right)\right)\right) \cos \left(\frac{p_{1}}{2} g_{1}\left(x_{1}\right)\right)(-1)^{\mathbf{x}+x_{1}}\left(-\partial_{1}^{2}\right) Y_{\varepsilon_{\Lambda}}(\mathbf{x})= \\
& =v_{1}^{2}|\Lambda|\left[e_{0}-\sin ^{2} \frac{p_{1}}{2} \sum_{\mathbf{x} \neq \mathbf{0}}\left(1-g_{0}\left(x_{1}\right)\right)(-1)^{\mathbf{x}+x_{1}}\left(-\partial_{1}^{2}\right) Y_{\varepsilon_{\Lambda}}(\mathbf{x})\right]
\end{aligned}
$$

The conclusion is

$$
\frac{1}{|\Lambda|} \sum_{\mathbf{x}, \mathbf{y} \in \Lambda}\left(u_{\mathbf{x}}^{1}\right)^{*} W_{11}^{0}(\mathbf{x}-\mathbf{y}) u_{\mathbf{y}}^{1}=\alpha \sin ^{2} \frac{p_{1}}{2}
$$

where $\alpha=\sum_{\mathbf{x} \neq \mathbf{0}}\left(1-g_{0}\left(x_{1}\right)\right)(-1)^{\mathbf{x}+x_{1}} \partial_{1}^{2} Y_{\varepsilon_{\Lambda}}(\mathbf{x})$. We will show in Appendix A that $\alpha>0$. Let us now consider $\sum_{\mathbf{x}, \mathbf{y} \in \Lambda}\left(u_{\mathbf{x}}^{2}\right)^{*} W_{22}(\mathbf{x}-\mathbf{y}) u_{\mathbf{y}}^{2}$. This double summation is equal to

$$
v_{2}^{2} \sum_{\mathbf{x} \in \Lambda} \sum_{\mathbf{y} \in \mathbb{Z}^{d} \backslash \mathbf{x}} e^{-i \frac{p_{1}}{2}\left(f_{1}\left(x_{1}\right)-f_{1}\left(y_{1}\right)\right)} f_{0}\left(x_{1}\right) f_{0}\left(y_{1}\right)(-1)^{\left(x_{3}-y_{3}\right)+\cdots+\left(x_{d}-y_{d}\right)}\left(-\partial_{2}^{2}\right) Y_{\varepsilon_{\Lambda}}(\mathbf{x}-\mathbf{y})
$$

Using that $f_{1}(x) f_{1}(y)=g_{0}(x-y)+(-1)^{x} g_{1}(x-y)$ and $f_{0}(x) f_{0}(y)=g_{0}(x-y)-(-1)^{x} g_{1}(x-y)$, we can rewrite this as:

$$
\begin{gathered}
v_{2}^{2} \sum_{\mathbf{x} \in \Lambda} \sum_{\mathbf{y} \in \mathbb{Z}^{d} \backslash \mathbf{x}} e^{-i \frac{p_{1}}{2}\left\{f_{1}\left(x_{1}\right)\left[1-g_{0}\left(x_{1}-y_{1}\right)\right]+f_{0}\left(x_{1}\right) g_{1}\left(x_{1}-y_{1}\right)\right\}}\left[g_{0}\left(x_{1}-y_{1}\right)-(-1)^{x_{1}} g_{1}\left(x_{1}-y_{1}\right)\right] . \\
\cdot(-1)^{\left(x_{3}-y_{3}\right)+\cdots+\left(x_{d}-y_{d}\right)}\left(-\partial_{2}^{2}\right) Y_{\varepsilon_{\Lambda}}(\mathbf{x}-\mathbf{y})
\end{gathered}
$$

Performing summation over $\mathbf{x}$ yields

$$
\begin{aligned}
v_{2}^{2}|\Lambda| \sum_{\mathbf{x} \neq \mathbf{0}} & {\left[g_{0}\left(x_{1}\right) \cos \left(\frac{p_{1}}{2}\left(1-g_{0}\left(x_{1}\right)\right)\right) \cos \left(\frac{p_{1}}{2} g_{1}\left(x_{1}\right)\right)-\right.} \\
& \left.-g_{1}\left(x_{1}\right) \sin \left(\frac{p_{1}}{2}\left(1-g_{0}\left(x_{1}\right)\right)\right) \sin \left(\frac{p_{1}}{2} g_{1}\left(x_{1}\right)\right)\right](-1)^{x_{3}+\cdots+x_{d}}\left(-\partial_{2}^{2}\right) Y_{\varepsilon_{\Lambda}}(\mathbf{x})
\end{aligned}
$$

This can be rewritten as

$$
v_{2}^{2}|\Lambda|\left[e_{0}-\cos ^{2} \frac{p_{1}}{2} \sum_{\mathbf{x} \neq \mathbf{0}}\left(1-g_{0}\left(x_{1}\right)\right)(-1)^{\mathbf{x}+x_{2}}\left(-\partial_{2}^{2}\right) Y_{\varepsilon_{\Lambda}}(\mathbf{x})\right]
$$

The conclusion is

$$
\frac{1}{|\Lambda|} \sum_{\mathbf{x}, \mathbf{y} \in \Lambda}\left(u_{\mathbf{x}}^{2}\right)^{*} W_{22}^{0}(\mathbf{x}-\mathbf{y}) u_{\mathbf{y}}^{2}=\gamma \cos ^{2} \frac{p_{1}}{2}
$$

where $\gamma=\sum_{\mathbf{x} \neq \mathbf{0}}\left(1-g_{0}\left(x_{1}\right)\right)(-1)^{\mathbf{x}+x_{2}} \partial_{2}^{2} Y_{\varepsilon_{\Lambda}}(\mathbf{x})$. We will show in Appendix A that $\gamma>0$. Finally (3.43) is valid even if in the l.h.s we exchange the index 2 with $j \geq 3$. Substituting all this into (3.32) yields:

$$
\hat{W}^{0}(\mathbf{p}) \geq\left(\begin{array}{cccc}
\alpha \sin ^{2} \frac{p_{1}}{2} & 0 & 0 & 0 \\
0 & \gamma \cos ^{2} \frac{p_{1}}{2} & 0 & 0 \\
0 & 0 & \ddots & 0 \\
0 & 0 & 0 & \gamma \cos ^{2} \frac{p_{1}}{2}
\end{array}\right) .
$$

By interchanging the roles of $p_{1}, \ldots, p_{d}$ we finally get

$$
\hat{W}^{0}(\mathbf{p}) \geq Y(\mathbf{p}), \quad Y_{i j}(\mathbf{p})=\frac{1}{d} \delta_{i j}\left(\alpha \sin ^{2} \frac{p_{i}}{2}+\gamma \sum_{l \neq i} \cos ^{2} \frac{p_{l}}{2}\right)
$$


This bound proves Conjecture 7.9 in [10].

Now, the proof of existence of long range order, given the bounds (3.30) and (3.45), is standard. We reproduce it here, for completeness. Given $\ell \in\{1, \ldots, d\}$, we note that, by the rotation symmetry of $\langle\cdot\rangle_{\beta, \Lambda}$,

$$
\frac{1}{|\Lambda|} \sum_{\mathbf{p}}\left\langle\hat{S}_{\mathbf{p}}^{\ell} \hat{S}_{-\mathbf{p}}^{\ell}\right\rangle_{\beta, \Lambda}=\frac{1}{d}
$$

Therefore,

$$
\frac{1}{|\Lambda|}\left\langle\hat{S}_{\boldsymbol{\pi}_{\ell}}^{\ell} \hat{S}_{-\boldsymbol{\pi}_{\ell}}^{\ell}\right\rangle_{\beta, \Lambda}=\frac{1}{d}-\frac{1}{|\Lambda|} \sum_{\mathbf{p} \neq \boldsymbol{\pi}_{\ell}} Q_{\ell \ell}(\mathbf{p}) .
$$

Let us now consider the sum in the r.h.s. Note that, for any $\mathbf{p} \notin S$, we can use (3.30) and (3.45) to conclude that

$$
Q_{\ell \ell}(\mathbf{p}) \leq \frac{d}{2 \beta}\left(\alpha \sin ^{2} \frac{p_{\ell}}{2}+\gamma \sum_{j \neq \ell} \cos ^{2} \frac{p_{j}}{2}\right)^{-1}
$$

On the other hand, for $\mathbf{p}=\boldsymbol{\pi}_{m}, m \neq \ell$, we can note that $\hat{W}_{i j}^{0}\left(\boldsymbol{\pi}_{m}\right)=\delta_{i j}\left(1-\delta_{i m}\right)\left(e_{1}-e_{0}\right)$, with $e_{1}=\sum_{\mathbf{x} \neq \mathbf{0}}(-1)^{\mathbf{x}+x_{2}}\left(-\partial_{1}^{2}\right) Y_{\varepsilon_{\Lambda}}(\mathbf{x})$ and $e_{0}$ defined in (3.21). Using (3.45), we see that $e_{1}-e_{0} \geq(\alpha+\gamma) / d$. Therefore, if $v^{i}=\delta_{i \ell} /\left(e_{1}-e_{0}\right)$, we can use (3.29) to get

$$
\sum_{i, j} v^{i}\left[\hat{W}^{0}\left(\boldsymbol{\pi}_{m}\right) Q\left(\boldsymbol{\pi}_{m}\right) \hat{W}^{0}\left(\boldsymbol{\pi}_{m}\right)\right]_{i j} v^{j}=Q_{\ell \ell}\left(\boldsymbol{\pi}_{m}\right) \leq \frac{1}{2 \beta} \frac{1}{e_{1}-e_{0}} \leq \frac{d}{2 \beta} \frac{1}{\alpha+\gamma}
$$

and we conclude that (3.48) is valid for $\mathbf{p}=\boldsymbol{\pi}_{m}, m \neq \ell$, as well. Substituting (3.48) we get

$$
\frac{1}{|\Lambda|}\left\langle\hat{S}_{\boldsymbol{\pi}_{\ell}}^{\ell} \hat{S}_{-\boldsymbol{\pi}_{\ell}}^{\ell}\right\rangle_{\beta, \Lambda} \geq \frac{1}{d}-\frac{d}{2 \beta|\Lambda|} \sum_{\mathbf{p} \neq \boldsymbol{\pi}_{\ell}} \frac{1}{\alpha \sin ^{2} \frac{p_{\ell}}{2}+\gamma \sum_{j \neq \ell} \cos ^{2} \frac{p_{j}}{2}} .
$$

If we note that

$$
\left\langle\hat{S}_{\boldsymbol{\pi}_{\ell}}^{\ell} \hat{S}_{-\boldsymbol{\pi}_{\ell}}^{\ell}\right\rangle_{\beta, \Lambda}=\frac{1}{|\Lambda|} \sum_{\mathbf{x}, \mathbf{y} \in \Lambda}\left\langle\sigma_{\mathbf{x}}^{\ell} \sigma_{\mathbf{y}}^{\ell}\right\rangle_{\beta, \Lambda}
$$

and take the thermodynamic limit in (3.50) we finally get (2.5), with

$$
c_{d}(\beta)=1-\frac{d^{2}}{2 \beta} \int_{[-\pi, \pi]^{d}} \frac{d \mathbf{p}}{(2 \pi)^{d}} \frac{1}{\alpha \sin ^{2} \frac{p_{\ell}}{2}+\gamma \sum_{j \neq \ell} \cos ^{2} \frac{p_{j}}{2}} .
$$

\section{Acknowledgments}

I am grateful to Joel L. Lebowitz and Elliott H. Lieb for stimulating my interest for this problem and for many helpful discussions. Part of this work was supported by the Department of Physics of Princeton University and by U.S. National Science Foundation grant PHY-0652854, which are gratefully acknowledged.

\section{APPENDIX A: THE CONSTANTS $\alpha$ AND $\gamma$ ARE POSITIVE}

In this Appendix we show that the constants $\alpha$ and $\gamma$ in (3.45), defined right after (3.38) and (3.43) respectively, are positive. Let us first consider $\alpha=\sum_{\mathbf{x} \neq \mathbf{0}}\left(1-g_{0}\left(x_{1}\right)\right)(-1)^{\mathbf{x}+x_{1}} \partial_{1}^{2} Y_{\varepsilon_{\Lambda}}(\mathbf{x})$. Note that $1-g_{0}\left(x_{1}\right)$ is a non-negative even function of $x_{1}$, vanishing at $x_{1}=0$. Using (3.12) we can rewrite

$$
\alpha=\frac{1}{(2 \pi)^{d-1}} \sum_{\substack{x_{1}>0 \\ \mathbf{x}_{\perp} \in \mathbb{Z}^{d-1}}}\left(1-g_{0}\left(x_{1}\right)\right) \int d \mathbf{k}_{\perp} \sqrt{\mathbf{k}_{\perp}^{2}+\varepsilon_{\Lambda}^{2}} e^{i\left[\mathbf{k}_{\perp}+\pi \mathbf{1}\right] \cdot \mathbf{x}_{\perp}} e^{-x_{1} \sqrt{\mathbf{k}_{\perp}^{2}+\varepsilon_{\Lambda}^{2}}}
$$


where $\pi \mathbf{1}$ is the $(d-1)$-dimensional vector with components all equal to $\pi$. The summation over $\mathbf{x}_{\perp}$ can be explicitly computed and produces a $(d-1)$-dimensional delta function $(2 \pi)^{d-1} \sum_{\mathbf{m} \in \mathbb{Z}^{d-1}} \delta\left(\mathbf{k}_{\perp}+\pi(2 \mathbf{m}+\mathbf{1})\right)$. So we find

$$
\alpha=\sum_{x_{1}>0} \sum_{\mathbf{m} \in \mathbb{Z}^{d}}\left(1-g_{0}\left(x_{1}\right)\right) \sqrt{\pi^{2}(2 \mathbf{m}+\mathbf{1})^{2}+\varepsilon_{\Lambda}^{2}} e^{-x_{1} \sqrt{\pi^{2}(2 \mathbf{m}+\mathbf{1})^{2}+\varepsilon_{\Lambda}^{2}}}>0
$$

and the proof that $\alpha>0$ is concluded.

Similarly, let us consider $\gamma=\sum_{\mathbf{x} \neq \mathbf{0}}\left(1-g_{0}\left(x_{1}\right)\right)(-1)^{\mathbf{x}+x_{2}} \partial_{2}^{2} Y_{\varepsilon_{\Lambda}}(\mathbf{x})$. Note that $(-1)^{x_{1}}(1-$ $\left.g_{0}\left(x_{1}\right)\right)$ is an even function of $x_{1}$, vanishing at $x_{1}=0$ with alternating signs. Using (3.12) we can rewrite

$$
\gamma=\frac{1}{(2 \pi)^{d-1}} \sum_{\substack{x_{1}>0 \\ \mathbf{x}_{\perp} \in \mathbb{Z}^{d-1}}}(-1)^{x_{1}+1}\left(1-g_{0}\left(x_{1}\right)\right) \int \frac{d \mathbf{k}_{\perp}}{\sqrt{\mathbf{k}_{\perp}^{2}+\varepsilon_{\Lambda}^{2}}} k_{2}^{2} e^{i k_{2} x_{2}} e^{-x_{1} \sqrt{\mathbf{k}_{\perp}^{2}+\varepsilon_{\Lambda}^{2}}} \prod_{j \geq 3} e^{i\left(k_{j}+\pi\right) x_{j}}
$$

Performing the summation over $\mathbf{x}_{\perp}$ and using that $(-1)^{x_{1}+1}\left(1-g_{0}\left(x_{1}\right)\right)=\{0,1,-2,1\}$, we find

$$
\gamma=\sum_{\mathbf{m} \in \mathbb{Z}^{d-1}} \frac{4 \pi^{2} m_{1}^{2}}{\sqrt{\pi^{2}\left(2 \mathbf{m}+\pi \mathbf{1}-\hat{e}_{1}\right)^{2}+\varepsilon_{\Lambda}^{2}}} \frac{\cosh \sqrt{\pi^{2}\left(2 \mathbf{m}+\pi \mathbf{1}-\hat{e}_{1}\right)^{2}+\varepsilon_{\Lambda}^{2}}-1}{\sinh 2 \sqrt{\pi^{2}\left(2 \mathbf{m}+\pi \mathbf{1}-\hat{e}_{1}\right)^{2}+\varepsilon_{\Lambda}^{2}}}
$$

where $\hat{e}_{1}=(1,0, \ldots, 0)$. The proof that $\gamma>0$ is concluded.

[1] G. Benfatto, G. Gallavotti, and F. Nicol'o: The dipole Phase in the Two-dimensional Hierarchical Coulomb Gas: Analyticity and Correlations Decay, Comm. Math. Phys. 106, 277-288 (1986).

[2] G. Benfatto, G. Gallavotti, and F. Nicol'o: On the Analyticity of the Pressure in the Hierarchical Dipole Gas, Jour. Stat. Phys. 55, 739-744 (1989).

[3] D. C. Brydges and Ph. A. Martin: Coulomb Systems at Low Density: A Review, Jour. Stat. Phys. 96, 1163-1330 (1999).

[4] D. C. Brydges and H. Yau: Grad $\phi$ perturbations of massless Gaussian fields, Comm. Math. Phys. 129, 351-392 (1990).

[5] A. Carbognani, E. Rastelli, S. Regina and A. Tassi: Dipolar interaction and long-range order in the square planar rotator model, Phys. Rev. B 62, 1015 (2000).

[6] K. De'Bell, A. B. MacIsaac, I. N. Booth, and J. P. Whitehead: Dipolar-induced planar anisotropy in ultrathin magnetic films, Phys. Rev. B 55, 15108 (1997).

[7] K. De'Bell, A. B. MacIsaac and J. P. Whitehead: Dipolar effects in magnetic thin films and quasi-two-dimensional systems, Rev. Mod. Phys. 72, 225 (2000).

[8] J. Frohlich, R. Israel, E. H. Lieb and B. Simon: Phase Transitions and Reflection Positivity. I. General Theory and Long Range Lattice Models, Comm. Math. Phys. 62, 1 (1978).

[9] J. Fröhlich and Y. M. Park: Correlation inequalities and the thermodynamic limit for classical and quantum continuous systems, Comm. Math. Phys. 59, 235-266 (1978).

[10] J. Fröhlich and T. Spencer: On the statistical mechanics of classical Coulomb and dipole gases, Jour. Stat. Phys. 24, 617-701 (1981).

[11] J. Fröhlich, B. Simon and T. Spencer: Infrared bounds, phase transitions and continuous symmetry breaking, Comm. Math. Phys. 50, 79-95 (1976).

[12] K. Gawedski and A. Kupiainen: Block spin renormalization group for dipole gas and $(\nabla \phi)^{4}$, Ann. Phys. (N. Y.) 147, 198-243 (1983).

[13] A. Giuliani, J. L. Lebowitz and E. H. Lieb: Striped phases in two dimensional dipole systems, Phys. Rev. B 76, 184426 (2007).

[14] A. Naddaf and T. Spencer: On homogenization and scaling limit of some gradient perturbations of a massless free field, Comm. Math. Phys. 183, 55-84 (1997).

[15] Y. M. Park: Lack of screening in the continuous dipole system, Comm. Math. Phys. 70, 161-167 (1979). 